\title{
Integration of fast fluid dynamics and Markov chain model for predicting transient particle transport in buildings
}

\author{
Wei $\mathrm{Liu}^{1, *}$, and Chun Chen ${ }^{2,3}$ \\ ${ }^{1}$ Division of Sustainable Buildings, Department of Civil and Architectural Engineering, KTH Royal Institute of Technology, \\ Brinellvägen 23, Stockholm, 100 44, Sweden \\ ${ }^{2}$ Department of Mechanical and Automation Engineering, The Chinese University of Hong Kong, Shatin, N.T. 999077, Hong Kong \\ SAR, China \\ ${ }^{3}$ Shenzhen Research Institute, The Chinese University of Hong Kong, Shenzhen 518057, China
}

\begin{abstract}
Fast simulation tools for the prediction of transient particle transport are critical in designing the air distribution indoors to reduce the exposure to indoor particles and associated health risks. This investigation proposed a combined fast fluid dynamics (FFD) and Markov chain model for fast predicting transient particle transport indoors. The solver for FFD-Markov-chain model was programmed in OpenFOAM, an open-source CFD toolbox. This study used a case from the literature to validate the developed model and found well agreement between the transient particle concentrations predicted by the FFD-Markov-chain model and the experimental data. This investigation further compared the FFD-Markovchain model with the CFD-Eulerian model and CFD-Lagrangian model in terms of accuracy and efficiency. The accuracy of the FFD-Markov-chain model was similar to that of the other two models. For the studied case, the FFD-Markov-chain model was 4.7 times faster than the CFD-Eulerian model, and it was 137.4 times faster than the CFD-Lagrangian model in predicting the steady-state airflow and transient particle transport. Therefore, the FFD-Markov-chain model is able to greatly reduce the computing cost for predicting transient particle transport in indoor environments.
\end{abstract}

\section{Introduction}

Exposure to outdoor particles has been linked to various adverse health effects, such as lung cancer [1] and shortened life expectancy [2]. Particles that originate outdoors can enter indoor environments through natural ventilation, mechanical ventilation, and infiltration [3]. As people spend most of their time indoors [4], their exposure to particles of outdoor origin occurs mainly indoors [5]. In addition to particles from the outdoor environment, there are many indoor particle sources, such as cigarette smoking [6], cooking [7], human activity [8], and chemical reactions [9]. Furthermore, particles exhaled by an infected person can result in the transmission of many airborne infectious diseases indoors, including influenza [10], tuberculosis [11], measles [12], and severe acute respiratory syndrome (SARS) [13]. In most cases, the particle concentration distribution indoors is non-uniform, and the transport process is transient in nature. Therefore, it is crucial to correctly predict the transient particle transport in indoor environments in order to improve the design of air distribution and reduce health risks.

Computational fluid dynamics (CFD) is among the most popular tools for modelling airflow distribution and particle transport. Among the numerical methods, fast fluid dynamics (FFD) and the Markov chain model can accelerate the airflow and transient particle transport calculations, respectively. However, the combined effects on accuracy and computing cost are unknown. Therefore, this study aimed to develop a combined FFD and Markov chain model and evaluate the model in terms of accuracy and computing cost.

\section{Methods}

\subsection{Fast fluid dynamics for airflow and turbulence}

This study considered the time-dependent Navier-Stokes equations for incompressible viscous indoor airflow:

$$
\begin{gathered}
\frac{\partial U_{i}}{\partial x_{i}}=0 \\
\frac{\partial U_{i}}{\partial t}+U_{j} \frac{\partial U_{i}}{\partial x_{j}}=v \frac{\partial^{2} U_{i}}{\partial x_{j} \partial x_{j}}-\frac{1}{\rho} \frac{\partial p}{\partial x_{i}}+\frac{1}{\rho} F_{i}
\end{gathered}
$$

where $U_{i}$ is the air velocity component in the $\mathrm{Xi}(\mathrm{i}=1,2,3)$ direction, $\mathrm{t}$ is the time, $v$ is the effective viscosity, $\mathrm{Q}$ is the density, and $\mathrm{F}_{\mathrm{i}}$ is the ith component of the body forces. A standard incremental pressure-correction (SIPC) scheme was applied to solve the Navier-Stokes

\footnotetext{
${ }^{*}$ Corresponding author: wei.liu@byv.kth.se
} 
equations. The SIPC scheme splits Eq. (2) into the following two equations:

$$
\begin{gathered}
\frac{U_{i}^{*}-U_{i}^{n}}{\Delta t}=-U_{j}^{n} \frac{\partial U_{i}^{*}}{\partial x_{j}}+v \frac{\partial^{2} U_{i}^{*}}{\partial x_{j} \partial x_{j}}-\frac{1}{\rho} \frac{\partial p^{n}}{\partial x_{i}}+\frac{1}{\rho} F_{i} \\
\frac{U_{i}^{n+1}-U_{i}^{*}}{\Delta t}=-\frac{1}{\rho} \frac{\partial\left(p^{n+1}-p^{n}\right)}{\partial x_{i}}
\end{gathered}
$$

The pressure projection method substitutes Eq. (4) into Eq. (1) to produce:

$$
\frac{\partial^{2}\left(p^{n+1}-p^{n}\right)}{\partial x_{i} \partial x_{i}}=\frac{\rho}{\Delta t} \frac{\partial U_{i}^{*}}{\partial x_{i}}
$$

The SIPC scheme first solves Eq. (3), which considers the advection and diffusion terms implicitly and the pressure and source terms explicitly. With the solved intermediate velocity, the SIPC scheme then solves Eq. (5) to obtain the pressure. Finally, the SIPC makes a correction to the air velocity using Eq. (4).

To predict the transport of energy, the corresponding scalar transport equation must be solved:

$$
\frac{\partial T}{\partial t}+U_{j} \frac{\partial T}{\partial x_{j}}=\kappa \frac{\partial^{2} T}{\partial x_{j} \partial x_{j}}+S_{T}
$$

where $T$ is the air temperature, $k$ is the effective thermal conductivity, and $S_{T}$ is the energy source. Since Eq. (6) is a linear partial differential equation, this study solved it them iteratively with implicit schemes. With the solution of Eq. (6), FFD adopts the Boussinesq approximation [14] to simulate the buoyancy effect in the indoor airflow.

This study used the renormalization group (RNG) k- $\varepsilon$ model to calculate airflow and turbulence, as this model has the best overall performance among all Reynoldsaveraged Navier-Stokes (RANS) models for enclosed environments [15].

\subsection{Markov chain model for transient particle transport}

The Markov chain model calculates the number of particles in a given cell (represented by the subscript $i$ ) at the subsequent time step $(t+\Delta t)$ on the basis of the Markov chain scheme [16]:

$$
N_{i}(t+\Delta t)=N_{i}(t) P_{i, i}+\sum_{n b} N_{n b}(t) P_{n b, i}
$$

where $N_{i}(t)$ represents the number of particles in cell $i$ at time $t$, the subscript $n b$ represents the neighboring cells or boundaries, $P_{i, i}$ is the probability of a particle's staying in cell $i$ in a certain time step $\Delta t$, and $P_{n b, i}$ is the probability of a particle's moving from a neighboring cell to cell $i$ in a certain time step $\Delta t$. Here, $P_{i, i}$ and $P_{n b, i}$ are called transition probabilities.

On the basis of the particle mass balance equation for the cell $i$, after a certain time step $\Delta t$, the probability of a particle's remaining in the current cell can be calculated by [16]:

$$
P_{i, i}=\exp \left(-\sum \frac{Q_{i, n b}}{V_{i}} \Delta t\right)
$$

If cell $j$ is one of the neighboring cells, the probability of a particle's moving from cell $i$ to cell $i$ in $\Delta t$ can be calculated by:

$$
P_{i, j}=\frac{Q_{i, j}}{\sum n b Q_{i, n b}}\left(1-P_{i, i}\right)
$$

where $Q_{i, n b}$ is the airflow rate from cell $i$ to the neighboring cell or boundary $n b$, which can be expressed as the sum of the mean airflow rate $Q_{\text {mean,i,nb }}$ and the turbulent fluctuating airflow rate $Q_{\text {fluctuating,inb. With the }}$ use of FFD simulation, the mean airflow rate can be obtained from the cell face air flux.

Please note that in Eq. (7), $P_{n b, i}$ is used instead of $P_{i, n b}$. Eq. (7) also shows that the Markov chain model does not require iterations in each time step, and thus the use of this model reduces the computing cost. The particle number concentration in cell $i$ at time $t$ can be calculated by:

$$
C_{i}(t)=\frac{N_{i}(t)}{V_{i}}
$$

where $V_{i}$ is the volume of the cell $i$.

This Markov chain model is effective for particles with a diameter smaller than $3 \mu \mathrm{m}$, which have negligible inertial effects [17]. Furthermore, when there is a constant particle source, to reduce the computing cost, the superimposition method can used as recommended by Gupta et al. [18].

\subsection{Implementation in OpenFOAM}

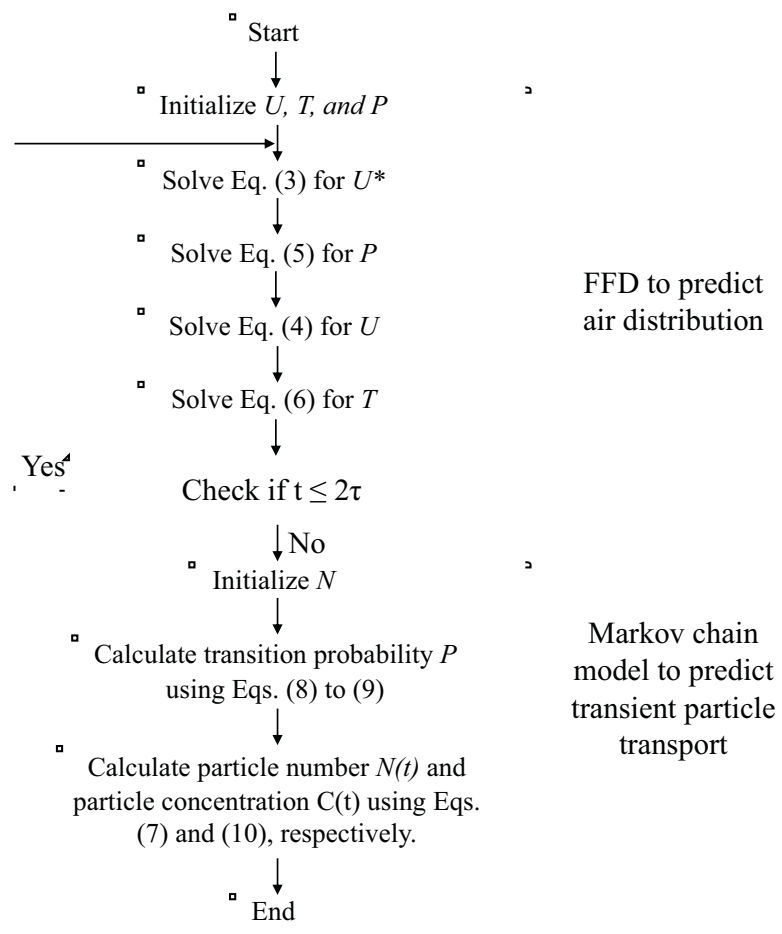

Fig. 1. Solution flow chart of the combined FFD and Markov chain model. 
This study implemented the FFD solver for air distribution and the Markov chain model for particle transport in OpenFOAM, an open-source CFD toolbox. Figure 1 shows the solution flow chart, where $\tau$ is the room time constant (s). The developed solver first conducts FFD simulation for $2 \tau$ to obtain a steady-state airflow, and then it calculates the transition probabilities, which are further used to calculate the number of particles at each time step.

\section{Validation and evaluation}

This investigation used a case, transient particle transport in an isothermal ventilated chamber, to validate the FFDMarkov-chain model. Next, the computing cost of the FFD-Markov-chain model was compared with the traditional CFD-Eulerian and CFD-Lagrangian models.

In Figure 2, the spatial dimensions of the room were $4 \mathrm{~m}$ in length, $2.1 \mathrm{~m}$ in width, and $2.4 \mathrm{~m}$ in height. There was an inlet $(0.3 \mathrm{~m} \times 0.3 \mathrm{~m})$ located $0.3 \mathrm{~m}$ below the ceiling on the left wall and an outlet $(0.3 \mathrm{~m} \times 0.3 \mathrm{~m})$ located $0.3 \mathrm{~m}$ above the floor on the right wall. Air was supplied with velocity magnitude of $0.84 \mathrm{~m} / \mathrm{s}$ and incident angle of 10 degree downward, and turbulence intensity of $20 \%$. Particles with a diameter of $1 \mu \mathrm{m}$ were injected into the chamber through the inlet. The experiment measured the transient particle concentrations at two locations on a vertical line in the $\mathrm{z}-$ directional center-cutting plane and $1 \mathrm{~m}$ away from the inlet. The measurement locations were $1.8 \mathrm{~m}$ (point 1) and $0.9 \mathrm{~m}$ (point 2) above the floor.

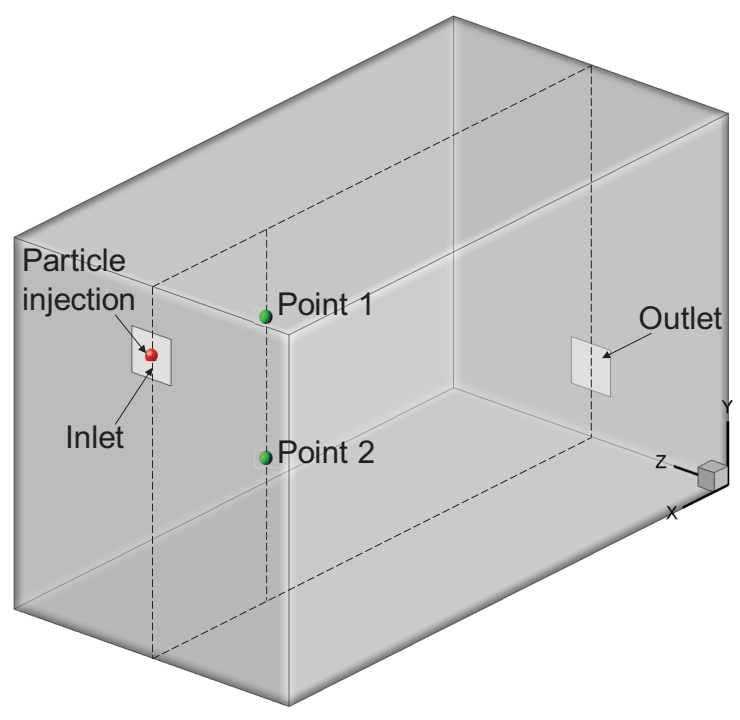

Fig. 2. Configuration of the chamber studied by Zhang et al. [21].

The numerical simulation used a grid with 18,009 hexahedral cells, on the basis of the grid independence test by Chen et al. [17]. The time step size for FFD was set at $1 \mathrm{~s}$, which was sufficiently fine to capture the flow features according to the independence test. The room time constant was 135 s. Therefore, the physical flow time for FFD calculation was set at $270 \mathrm{~s}$. The time step size for the Markov chain model was set at $0.01 \mathrm{~s}$. The physical flow time was set at 1,500 s, as all of the particles were removed from the room after 1,500 s. The influence of particle deposition and resuspension were negligible when the particle size was $1 \mu \mathrm{m}$ [19].

Figure 3 compares the numerical results for the transient particle concentrations with the experimental data. The numerical results obtained with the CFDEulerian and CFD-Lagrangian models [20] are also included for comparison. Note that the three sets of simulations were performed on the basis of the same inlet particle concentrations, as shown in Figure 5 of Zhang et al. [21]. The FFD-Markov-chain model correctly predicted the strong initial peak and the relatively weak and delayed peak in particle concentrations at the height of 1.8 and $0.9 \mathrm{~m}$ in Figures 3(a) and 3(b), respectively. The curve predicted by the FFD-Markov-chain model agreed well with the experimental data, as shown in Figure 3(a). However, as displayed in Figure 3(b), the FFD-Markov-chain model over-predicted the particle concentration after the peak in comparison with the experimental data. Interestingly, the over-prediction also occurred for the CFD-Eulerian and CFD-Lagrangian models. The FFD-Markov-chain model at least achieved similar accuracy to that of the other two models. The discrepancies between the simulations and experiment may be partially attributed to heat generation by the particle counters and the associated convective flow. In general, the accuracy of the FFD-Markov-chain model was satisfactory.

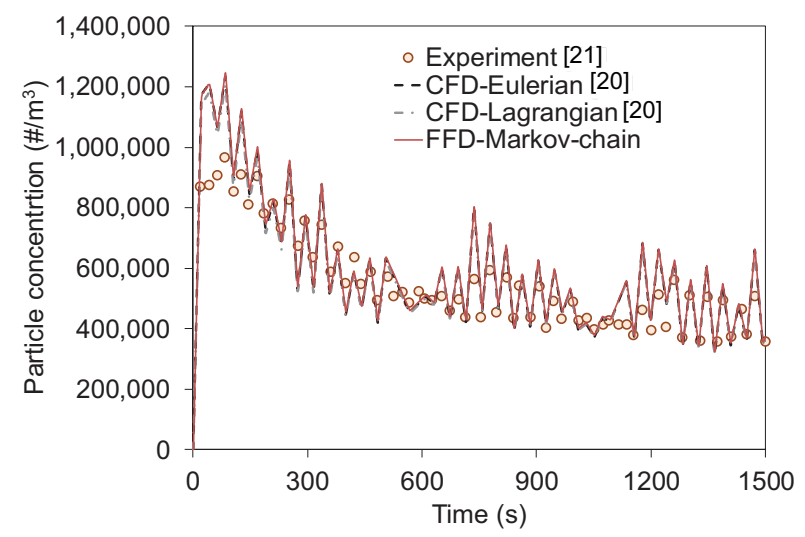

(a)

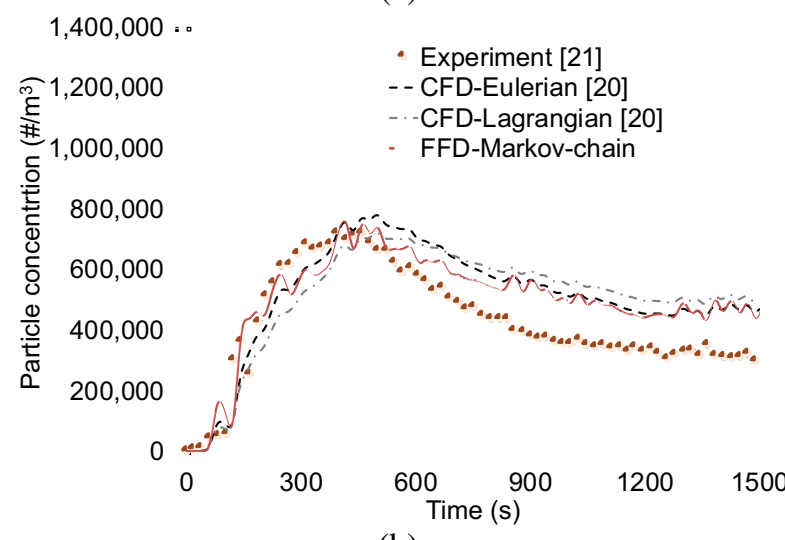

(b) 
Fig. 3. Comparison of the numerical results for transient particle concentration with the corresponding experimental data: (a) $\mathrm{y}=1.8 \mathrm{~m}$, (b) $\mathrm{y}=0.9 \mathrm{~m}$.

\section{Comparison of computing costs}

The validation cases above have shown that the FFDMarkov-chain model can predict indoor transient particle transport reasonably well, with accuracy similar to that of the CFD-Eulerian and CFD-Lagrangian models. This section discusses another important factor, the computing cost of the FFD-Markov-chain model. Note that the calculations were performed using the same computer under the same conditions. Figure 6 compares the total computing time for the FFD-Markov-chain model with the times required by the CFD-Eulerian and CFD-Lagrangian models. For the case, the FFD-Markovchain model took $3.6 \mathrm{~min}$, while the CFD-Eulerian and CFD-Lagrangian models took 20.7 and $501.5 \mathrm{~min}$, respectively. Namely, the FFD-Markov-chain model was 4.7 and 137.4 times faster, respectively, than the CFDEulerian and CFD-Lagrangian models.

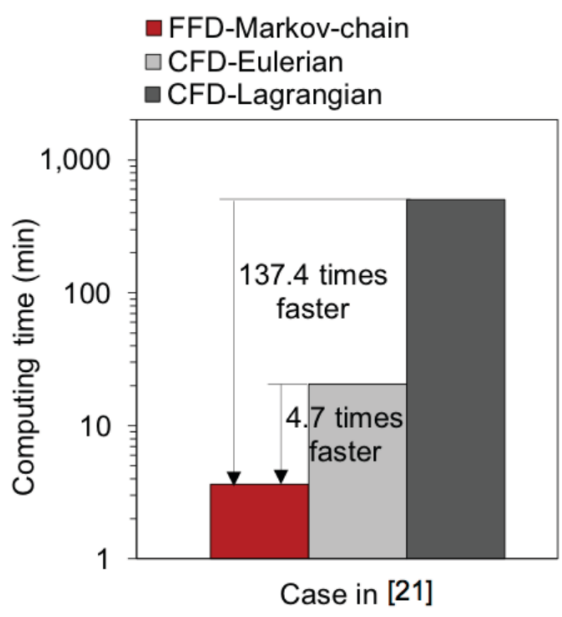

Fig. 4. Comparison of the computing time of the FFD-Markovchain model with the computing times of the CFD-Eulerian and CFD-Lagrangian models.

Table 1. Comparison of computing time for airflow and particle transport calculations.

\begin{tabular}{|c|l|l|l|}
\hline Case & Model & $\begin{array}{l}\text { Computing } \\
\text { time for } \\
\text { airflow } \\
\text { (min) }\end{array}$ & $\begin{array}{l}\text { Computing } \\
\text { time for } \\
\text { particle } \\
\text { transport } \\
\text { (min) }\end{array}$ \\
\hline \multirow{2}{*}[21]{} & $\begin{array}{l}\text { FFD-Markov- } \\
\text { chain }\end{array}$ & 0.3 & 3.3 \\
\cline { 2 - 4 } & CFD-Eulerian & 2.2 & 18.5 \\
\cline { 2 - 4 } & CFD-Lagrangian & 2.2 & 499.4 \\
\hline
\end{tabular}

Table 1 further compares the computing time for the airflow and particle transport calculations. For the airflow calculations, FFD was 5.9 times faster than CFD for the case. For particle transport, the Markov chain model was 4.6 times faster than the Eulerian model for the case, and 149.7 times faster than the Lagrangian model for the case. In terms of absolute contribution to computing-time saving, the Markov chain model contributed more than FFD, as the transient particle transport calculations required more time than the airflow calculations. In general, the FFD-Markov-chain model significantly reduced the computing cost in comparison with the traditional CFD-Eulerian and CFDLagrangian models.

\section{Discussions}

There are several limitations of the FFD-Markov-chain model, starting with FFD. This study used the RNG k- $\varepsilon$ model in FFD to simulate the turbulence. For simulating more complicated flow such as indoor airflow in an aircraft cabin or outdoor airflow, large-eddy simulation (LES) would be a good choice. However, the integration of LES into FFD has not yet been achieved, and its performance in terms of accuracy and efficiency is unknown. In regard to the Markov chain model, the current version is not able to account for particle deposition, sedimentation, resuspension, coagulation, evaporation, or biological and chemical reaction. If the model is to be applied to more complex indoor environments, these influencing factors should be considered. The implementation of additional functions in the Markov chain model would allow it to compete with the well-developed Eulerian and Lagrangian models.

\section{Conclusions}

This study developed a combined FFD and Markov chain model for predicting transient particle transport in indoor environments. The FFD-Markov-chain model was implemented in OpenFOAM, an open-source CFD toolbox. A case with experimental data from the literature was used to validate the model. The accuracy and computing cost of the FFD-Markov-chain model were evaluated by comparing it with the traditional CFD-Eulerian and CFD-Lagrangian models. The FFDMarkov-chain model can predict transient particle transport in indoor environments reasonably well in comparison with experimental data. The accuracy of the FFD-Markov-chain model was similar to that of the traditional CFD-Eulerian and CFD-Lagrangian models. The FFD-Markov-chain model was 4.7 times faster than the CFD-Eulerian model. The FFD-Markov-chain model was 137.4 times faster than the CFD-Lagrangian model.

\section{Acknowledgement}

This work was partially supported by the project \#RNEp1-18 of the Shun Hing Institute of Advanced Engineering, The Chinese University of Hong Kong, and the National Natural Science Foundation of China (Grant No. 51808487). 


\section{References}

1. C.A. Pope, R.T. Burnett, M.J. Thun, E.E. Calle, D. Krewski, K. Ito, G.D. Thurston, Lung cancer, cardiopulmonary mortality, and long-term exposure to fine particulate air pollution, JAMA. 287 (2002) 1132-1141.

2. C.A. Pope, M. Ezzati, D.W. Dockery, Fineparticulate air pollution and life expectancy in the United States, N. Engl. J. Med. 360 (2009) 376-386.

3. C. Chen, B. Zhao, Review of relationship between indoor and outdoor particles: I/O ratio, infiltration factor and penetration factor, Atmos. Environ. 45 (2011) 275-288.

4. N.E. Klepeis, W.C. Nelson, W.R. Ott, J.P. Robinson, A.M. Tsang, P. Switzer, J.V. Behar, S.C. Hern, W.H. Engelmann, The National Human Activity Pattern Survey (NHAPS): a resource for assessing exposure to environmental pollutants, J. Expo. Anal. Env. Epid. 11 (2001) 231-252.

5. C. Chen, B. Zhao, C.J. Weschler, Indoor exposure to "outdoor PM10": assessing its influence on the relationship between PM10 and short-term mortality in US cities, Epidemiology. 23 (2012) 870-878.

6. E.D.L. Patino, J.A.Siegel, Indoor environmental quality in social housing: A literature review, Build. Environ. 131 (2018) 231-241.

7. Y. Zhao, B. Zhao, Emissions of air pollutants from Chinese cooking: A literature review, Build. Simul., doi.org/10.1007/s12273-018-0456-6.

8. R. You, W. Cui, C. Chen, B. Zhao, Measuring the short-term emission rate of particles in the "personal cloud" with different clothes and activity intensities in a sealed chamber, Aerosol Air Qual Res, 13 (2013) 911-921.

9. M. Yao, B. Zhao, SOA in newly decorated residential buildings, Build. Environ. 111 (2017) 132-139.

10. M.R. Moser, T.R. Bender, H.S. Margolis, G.R. Noble, A.P. Kendal, D.G. Ritter, An outbreak of influenza aboard a commercial airliner, Am. J. Epi. 110 (1979) 1-6.

11. D. Menzies, A. Fanning, L. Yuan, J.M. FitzGerald, Hospital ventilation and risk for tuberculous infection in Canadian health care workers, Ann. Int. Med. 133 (2000) 779-789.

12. A.B. Bloch, W.A. Orenstein, W.M. Ewing, W.H. Spain, G.F. Mallison, K.L. Herrmann, A.R. Hinman, Measles outbreak in a pediatric practice: Airborne transmission in an office setting, Pediatrics 75 (1985) 676-683.

13. I.T. Yu, Y. Li, T.W. Wong, W. Tam, A.T. Chan, J.H. Lee, D.Y. Leung, T. Ho, Evidence of airborne transmission of the severe acute respiratory syndrome virus, N. Engl. J. Med. 350 (2004) 17311739.

14. J. Boussinesq, Théorie analytique de la chaleur: mise en harmonie avec la thermodynamique et avec la théorie mécanique de la lumière. Vol. 2. Paris: Gauthier-Villars. (1903).

15. Z. Zhang, W. Zhang, Z. Zhai, Q. Chen, Evaluation of various turbulence models in predicting airflow and turbulence in enclosed environments by CFD: Part 2-Comparison with experimental data from literature, HVAC\&R Res. 13 (2007) 871-886.

16. M. Yao, B. Zhao, SOA in newly decorated residential buildings, Build. Environ. 111 (2017) 132-139.

17. C. Chen, W. Liu, C.-H. Lin, Q. Chen, A Markov chain model for predicting transient particle transport in enclosed environments, Build. Environ. 90 (2015) 30-36.

18. J.K. Gupta, C.H. Lin, Q. Chen, Inhalation of expiratory droplets in aircraft cabins. Indoor Air, 21 (2011) 341-350.

19. B. Zhao, C. Chen, Z. Tan, Modeling of ultrafine particle dispersion in indoor environments with an improved drift flux model, J. Aerosol Sci. 40 (2009) 29-43.

20. C. Chen, W. Liu, C.-H. Lin, Q. Chen, Comparing the Markov chain model with the Eulerian and Lagrangian models for indoor transient particle transport simulations, Aerosol Sci. Technol. 49 (2015) 857-871.

21. N. Zhang, Z. Zheng, S. Eckels, V.B. Nadella, X. Sun, Transient response of particle distribution in a chamber to transient particle injection. Part. Part. Syst Charact. 26 (2009) 199-209. 